\title{
Verbände-Anhörung zum Selbstverwaltungsstärkungsgesetz
}

Bei der Anhörung für das „Gesetz zur Stärkung der Handlungsfähigkeit der Selbstverwaltung der Spitzenorganisationen in der GKV“, kurz: GKV-Selbstverwaltungsstärkungsgesetz, Mitte Oktober im Bundesministerium für Gesundheit (BMG) haben die Verbandsvertreter aus dem Gesundheitswesen durchweg harsche Kritik gewählt. Geschlossen wurde der Vorwurf laut, dass das geplante Gesetz die Misstrauenskultur gegenüber der Selbstverwaltung offenlege.

In ihrer Ablehnung des geplanten Gesetzes war sich die Zahnärzteschaft - wie bereits bei der Hauptversammlung des Freien Verbandes Deutscher Zahnärzte (FVDZ) - einig (mehr dazu ab Seite 18). Die Kassenzahnärztliche Bundesvereinigung (KZBV) zeigte sich davon überzeugt, dass das Gesetz der Anfang vom Ende eines selbstverwalteten Gesundheitssystems sei. Der KZBVVorstandsvorsitzende Dr. Wolfgang Eßer forderte Bundesgesund-

Kassenärztliche Bundesvereinigung

\section{Flut von Vorwürfen gegen KBV-Chef}

Die Kassenärzte kommen nicht zur Ruhe: Wie kürzlich bekannt wurde, hat die stellvertretende Vorstandsvorsitzende Regina Feldmann vom Bundesministerium für Gesundheit (BMG) ein Verfahren wegen Untreue gegen den Vorsitzenden der Kassenärztlichen Bundesvereinigung (KBV) Dr. Andreas Gassen verlangt. Nach einem Bericht der Frankfurter Allgemeinen Zeitung (FAZ) kritisierte Feldmann, dass Gassen im Zusammenhang mit den Regelverstößen des früheren KBV-Chefs Dr. Andreas Köhler keine Konsequenzen drohten.

Feldmann beruft sich auf ein Gutachten einer Anwaltskanzlei für das BMG. Dort heiße es, Gassen habe als Vorsitzender der KBV-Vertreterversammlung umstrittene Verträge rund um die überhöhten Pensionszahlungen Köhlers selbst unterzeichnet. Mit Blick auf das Gutachten verwies das BMG darauf, dass keine abschließenden Feststellungen getroffen werden können, weil die zur Aufklärung beauftragte Kanzlei keine internen Untersuchungen durchführen konnte. Diese Antwort des Ministeriums genügte Feldmann nicht. Sie zog daraufhin ihre Unterschrift unter zwei Vereinbarungen zwischen BMG und KBV zurück. Darin geht es der $F A Z$ zufolge um die Klärung und Abwicklung eines umstrittenen Immobiliengeschäftes rund um den Umzug der Körperschaft von Köln nach Berlin.

Danach berichtete das Handelsblatt von neuen Ungereimtheiten. Demnach habe der KBV-Chef eigenmächtig rund 40.000 Euro für eine politische Beratungsfirma ausgegeben. Die Firma habe Gassen im öffentlichen Umgang mit den Skandalen in der Körperschaft beraten. Laut Handelsblatt liegen jedoch für diesen Auftrag keine Verträge vor. Zudem stimmten die abgerechneten Reisekosten nicht mit den Belegen überein, und es wurden keine prüfbaren Leistungsbeschreibungen vorgelegt. Gassen wies die Vorwürfe umgehend zurück. Er habe das Vergabeverfahren selbst durch eine Wirtschaftsprüfungsgesellschaft untersuchen lassen. heitsminister Hermann Gröhe (CDU) auf, zu einer Rechtsaufsicht mit Außenmaß zurückzukehren.

Der FVDZ-Bundesvorsitzende Harald Schrader machte gegenüber dem Ministerium deutlich, dass auch der Freie Verband die subsidiäre Selbstverwaltung befürworte und unterstütze. Der Verband mache sich gemeinsam mit der KZBV für eine Qualifizierungskampagne für ehrenamtlich engagierte Zahnärzte in den Vertreterversammlungen der Kassenzahnärztlichen Vereinigungen stark, um auf dem hohen professionellen Niveau der zahnärztlichen Selbstverwaltung mitarbeiten zu können. „Die Regelungen, Abstimmungsvorbehalte und Eingriffe in den Haushalt, die in dem Gesetz festgeschrieben werden sollen, führen dazu, dass niemand mehr für diese wichtigen Gremien arbeiten möchte“, sagte Schrader. Eine Selbstverwaltung werde damit ad absurdum geführt.

cas/sas

\section{Vorwurf des TK-Chefs

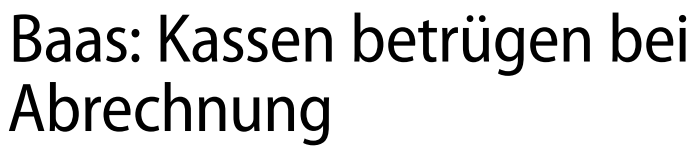

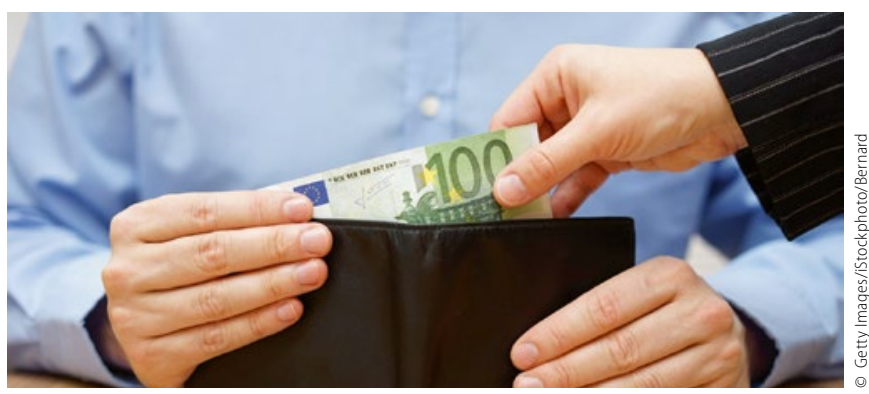

Krankenkassen bezahlen Ärzte dafür, dass sie falsche Diagnosen stellen, um mehr Geld einzunehmen. Dies erklärte der Vorstandsvorsitzende der Techniker Krankenkasse (TK), Jens Baas, in einem Interview mit der Frankfurter Allgemeinen Sonntagszeitung. „Die Kassen bezahlen zum Beispiel Prämien von zehn Euro je Fall für Ärzte, wenn sie den Patienten auf dem Papier kränker machen“, sagte der TK-Chef. Er begründete das Vorgehen damit, dass Kassen dann mehr Geld über den Risikostrukturausgleich (RSA) erhalten. Der RSA zahlt Kassen mit besonders kranken Versicherten einen Ausgleich. Durch diese Methoden nehmen die regionalen Kassen dem TK-Chef zufolge im laufenden Jahr eine Milliarde Euro mehr ein, als sie für die Versorgung der Versicherten benötigen. Baas räumte in dem Interview ein, dass sich auch seine Kasse „dem nicht völlig entziehen“ könne.

Die Reaktionen von anderen Kassen und aus der Ärzteschaft ließen nicht lange auf sich warten. Die Barmer GEK und die DAK-Gesundheit widersprachen den Vorwürfen vehement. Das Bundesversicherungsamt kritisierte den mutmaßlichen Abrechnungsbetrug durch die Kassen scharf und kündigte an, jedes Fehlverhalten konsequent zu verfolgen. Die Deutsche Stiftung Patientenschutz stellte sogar Strafanzeige gegen die TK und weitere Kassen. 